\title{
Enfermedad coronaria aneurismática severa en un adolescente con angina de esfuerzo secundaria a enfermedad de Kawasaki no diagnosticada previamente
}

\author{
Rienzi A. Díaz, Ernesto Aránguiz, 0 neglio Pedemonte, \\ D anilo Silva, Salvador Villablanca, H umberto Torres, \\ G ustavo Ríos.
}

\section{Severe aneurismal coronary artery disease probably caused by Kawasaki disease. Report of one case}

We report a 16 year old male with a history of angina on exertion. A treadmill exercise test was positive for ischemia in concordance with a Thallium-201 scintigraphy showing a septal and infero-posterior reversible myocardial perfusion defect. Coronary angiography disclosed severe aneurysmal coronary artery disease. Bilateral internal mammary coronary artery bypass grafting was successfully performed. Kawasaki disease is the most likely etiology, although not confirmed (Rev Méd Chile 2007; 135: 1182-85).

(Key words: Coronary aneurism; Coronary artery bypass grafting; Kawasaki disease)

Recibido el 4 de diciembre, 2006. Aceptado el 12 de diciembre, 2006.

Departamento de Cardiología, Clínica Reñaca, Cátedra de Cardiología, Universidad de Valparaíso, Chile.

L a angina de esfuerzos y los aneurismas de las _arterias coronarias son inhabituales en la gente joven. A su vez, el aneurisma coronario es poco frecuente en la población adulta, siendo su causa habitual la enfermedad coronaria ateroescleróti-

Correspondencia a: Dr. Rienzi A. Díaz. Clínica Reñaca. Casilla 9138, Correo 3. 4 Poniente 332, Viña del Mar, Chile. Teléfono: 56-32-2658581. Fax: 56-32-2658546. E mail: cardio@entelchile.net $\mathrm{ca}^{1}$. En los jóvenes, por el contrario, las lesiones coronarias aneurismáticas son por lo general secundarias a enfermedad de Kawasaki ${ }^{2}$.

Presentamos el caso de un adolescente hombre, con historia de angina de esfuerzo producto de enfermedad coronaria aneurismática, cuya etiología más probable es la enfermedad de Kawasaki.

\section{CASO CLÍNICO}

Comunicamos el caso de un adolescente de 16 años, estudiante secundario, previamente asinto- 
mático, que consultó en el Centro Médico de Clínica Reñaca con historia de angina de esfuerzos mayores, de varias semanas de evolución. El examen físico fue normal. Un electrocardiograma de reposo y una radiografía de tórax, fueron normales. Un test de esfuerzo bajo protocolo de Bruce, provocó a los $9 \mathrm{~min}$ un descenso del segmento ST de $2 \mathrm{~mm}$ en las derivaciones $A V_{\mathrm{L}}$ II, III, $\mathrm{AV}_{\mathrm{F}}, \mathrm{V}_{4}, \mathrm{~V}_{5}, \mathrm{~V}_{6}$. Una cintigrafía miocárdica con Talio-201, objetivó un defecto de perfusión posteroinferior y septal reversible, sugiriendo isquemia. Un ecocardiograma bidimensional mostró la existencia de una dilatación sacular de $7 \mathrm{~mm}$ en la porción proximal de la arteria coronaria izquierda (Figura 1-izquierda). Posteriormente, se realizó una coronariografía que demostró la existencia de enfermedad coronaria severa con dilatación aneurismática del segmento proximal de la arteria descendente anterior (Figura 1-centro) y el segmento proximal de la arteria coronaria derecha (Figura 2-izquierda), consistente con el aspecto angiográfico que se observa en la enfermedad de Kawasaki ${ }^{2}$, así como, la posterior oclusión de ambas arterias. Los dos vasos tenían llene a través de colaterales desde la arteria circunfleja y la primera diagonal. La ventriculografía izquierda fue normal.

El paciente no tenía antecedentes de dislipidemia, historia familiar de cardiopatía o trauma torácico. Sin embargo, a los 5 años de edad fue hospitalizado en una Unidad de Cuidados Intensivos Pediátricos de la $\mathrm{V}$ región, por un cuadro de fiebre alta, linfadenopatía cervical bilateral e insuficiencia cardíaca congestiva. Durante una prolongada hospitalización, requirió terapia con una combinación de drogas vasoactivas (dopamina, dobutamina, amrinona), así como ventilación mecánica. En la oportunidad, se planteó el diagnóstico de un síndrome de shock tóxico».

Otros estudios realizados con resultado negativo incluyeron: serología para sífilis, hepatitis $\mathrm{B}$ y C, ANCA, anticuerpos antinucleares y anticardiolipinas, así como, estudio de ultrasonido en búsqueda de aneurismas periféricos.

El paciente fue sometido a cirugía de revascularización coronaria con un puente secuencial de arteria mamaria interna izquierda a la arteria descendente anterior y primera diagonal, y un puente de arteria mamaria interna derecha a la arteria descendente posterior. Durante el acto quirúrgico, los hallazgos angiográficos previamente descritos fueron confirmados (Figura 1-derecha y Figura 2-derecha).

A 5 años de su seguimiento, el paciente está asintomático en tratamiento con aspirina $100 \mathrm{mg}$ al día, y un test de esfuerzo y cintigrafía miocárdica con Talio-201, resultaron normales.

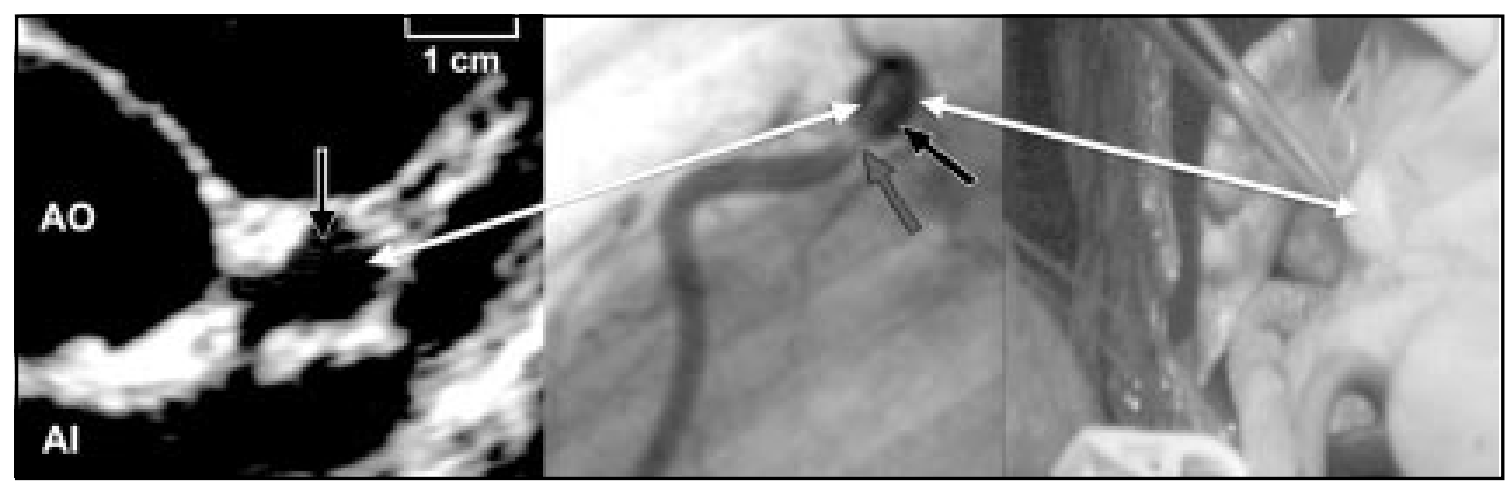

Figura 1. Izquierda, ecocardiograma bidimensional, eje corto, en el que se observa la aorta (AO) y el nacimiento de la arteria coronaria izquierda, que exhibe un aneurisma (flecha negra). Centro, coronariografía izquierda selectiva que muestra el aneurisma coronario con oclusión total de la arteria descendente anterior (flecha negra) y estenosis crítica de la primera diagonal (flecha gris). Derecha, hallazgo intraoperatorio que confirma el aneurisma de la arteria coronaria izquierda en concordancia con la imagen ecocardiográfica y angiográfica (flechas blancas). AI, aurícula izquierda. 


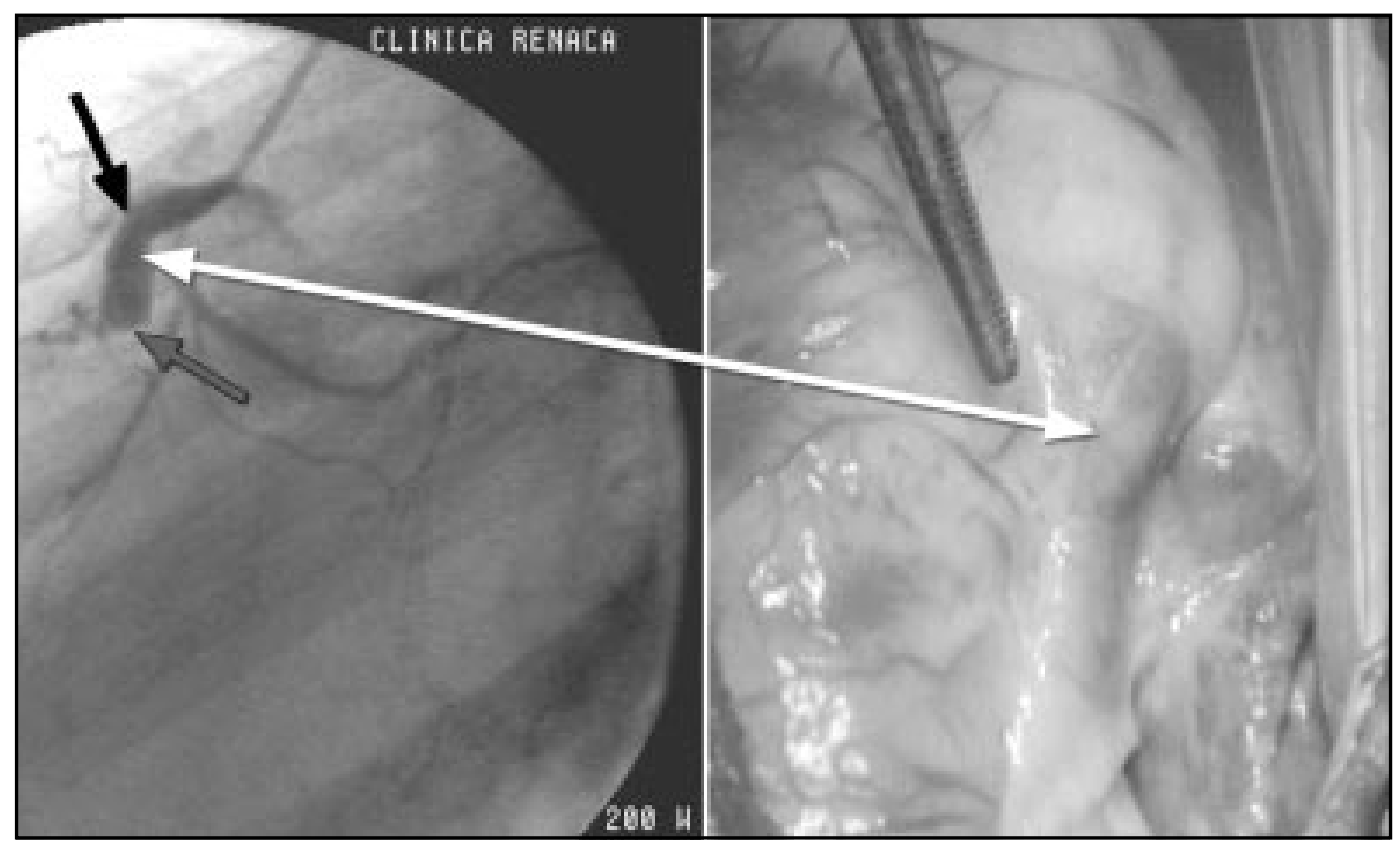

Figura 2. Izquierda, coronariografía derecha selectiva que muestra una dilatación aneurismática del tercio proximal de la arteria coronaria derecha (flecha negra) y luego su oclusión total (flecha gris). Derecha, hallazgo intraoperatorio que confirma el aneurisma de la arteria coronaria derecha, en concordancia con la imagen angiográfica (flecha blanca).

\section{Discusión}

En 1967, el Dr. Kawasaki (oftalmólogo en Japón) describió por vez primera lo que él denominó síndrome linfático nodular mucocutáneo, actualmente conocido como enfermedad de Kawasaki ${ }^{3}$. Esta entidad, es una vasculitis generalizada de origen desconocido que produce miocarditis y angitis. Esta última, se considera la causa de la formación de aneurismas en las arterias coronarias. La mayoría de los aneurismas que se forman, a pesar de la terapia con aspirina e inmunosupresores, permanecen silentes hasta la adolescencia 0 adultez temprana, período en el cual la manifestación principal es la angina de esfuerzo ${ }^{2}$. La historia y el cuadro clínico de nuestro paciente, permiten plantear la existencia de una enfermedad de Kawasaki previamente no diagnosticada, la que sólo fue descubierta luego del desarrollo de enfermedad coronaria oclusiva avanzada, que provocó la aparición de angina de esfuerzo. Lo anterior, es altamente probable ante la ausencia de factores de riesgo coronario, test serológicos negativos, inexistencia de traumatismo torácico y el descubrimiento de aneurismas coronarios típicos con enfermedad coronaria obstructiva severa, estos últimos hallazgos observados en la enfermedad de Kawasaki².

A su vez, nuestra hipótesis se refuerza por el antecedente de que nuestro paciente permaneció hospitalizado a la edad de 5 años, por un cuadro de fiebre que se asoció a disfunción miocárdica severa, esta última muy probablemente secundaria a miocarditis, la que junto a los aneurismas coronarios son dos de las principales manifestaciones de la enfermedad de Kawasaki. En ese momento, el diagnóstico planteado fue el de un síndrome de shock tóxico, cuadro clínico con el que la enfermedad de Kawasaki puede ser confundida ${ }^{4}$, como pensamos ocurrió en nuestro paciente.

Las indicaciones para la revascularización quirúrgica en la enfermedad coronaria, secundaria a la enfermedad de Kawasaki, son las mismas que 
para la enfermedad coronaria ateroesclerótica ${ }^{5}$. La angioplastia quirúrgica ${ }^{6}$ de los aneurismas es una alternativa terapéutica pero sólo para aneurismas gigantes (diámetros $>8 \mathrm{~mm})^{7}$. La angioplastia coronaria, puede ser considerada en algunos casos $^{8}$, pero fue descartada en nuestro paciente.

\section{REFERENCIAS}

1. Swaye PS, Fisher LD, Lutwin P, Vignola Pa, Judkins MP, KeMP HD ET AL. Aneurysmal coronary artery disease. Circulation 1983; 67: 134-8.

2. Burns JC, Shike H, Gordon JB, Malhotra A, SchoenwetTer M, Kawasaki T. Sequelae of Kawasaki disease in adolescents and young adults. J Am Coll Cardiol 1996; 28: 253-7.

3. KaWASAKI T. Acute febrile mucocutaneous syndrome with lymphoid involvement with specific desquamation of the fingers and toes in children. Jpn J Allergy 1967; 16: 178-222.

4. Spentzas T. Case 1. Toxic shock syndrome. Pediatrics in Review 1999; 20: 199-201.

5. Kttamura S, Kawashi K, Seki T, Morita R, Nishi T,
El presente caso, enfatiza que la enfermedad de Kawasaki debe ser considerada en pacientes jóvenes con cardiopatía isquémica asociada a aneurismas coronarios, en ausencia de factores de riesgo coronario.

MizUGUCHI K ET AL. Bilateral internal mammary artery grafts for comnary artery bypass operations in children. J Thorac Cardiov Surg 1990; 99: 708-15.

6. Pretre R, Turina MI. Surgical angioplasty of the left main coronary artery in non-atherosclerosis lesions. Heart 2000; 83: 91-3.

7. Brogan PA, Bose A, Burgner D, Shingadia D, TuLoh S, Michie C Eт al. Kawasaki disease: an evidence based approach to diagnosis, treatment, and proposals for the future research. Arch Dis Child 2002; 86: 286-90.

8. Ino T, Акімото K, Онкuвo M, Nishimoto K, Yabuta $\mathrm{K}$, TAKAYA J et aL. Application of percutaneous transluminal coronary angioplasty to coronary arterial stenosis in Kawasaki disease. Circulation 1996; 93: 1709-15. 